\title{
Evaluation of transrectal ultrasound-based dosimetry for brachytherapy of prostate cancer: a single-center experience
}

\author{
Changzhao Yang, MD', Zhengtong Lv, MD', Lingxiao Chen, MD', Jie Wang, MD², Xiheng Hu, MD!, \\ Harripersaud Chand, MD', Xi Sun, MD', Guyu Tang, MD', Congyi Tang, MD', Huichuan Jiang, MD', Yuan Li, MD' \\ 'Department of Urology, Xiangya Hospital, Central South University, Changsha, City. Hunan Province, P. R. China, ${ }^{2}$ Department of Radiotherapy, \\ Hunan Cancer Hospital, Changsha City, Hunan Province, P. R. China
}

\begin{abstract}
Purpose: To explore the possibility of intraoperative transrectal ultrasound (TRUS)-based dose verification in transperineal brachytherapy (BT) with iodine-125 $\left({ }^{125} \mathrm{I}\right)$ seeds for prostate cancer.

Material and methods: Fifteen patients with prostate cancer were treated using BT with ${ }^{125}$ I seeds. Post-implant TRUS and computed tomography (CT) images were imported into treatment planning system (TPS) for dosimetry. Dosimetry parameters, including minimum dose received by $90 \%$ of the volume $\left(D_{90}\right)$, percentage of the volume receiving $100 \%$ of prescribed dose $\left(\mathrm{V}_{100}\right)$, and percentage of the volume receiving $200 \%$ of prescribed dose $\left(\mathrm{V}_{200}\right)$ were calculated based on TRUS and CT images, separately. The $\mathrm{D}_{90}$ value of TRUS-based dosimetry was transformed to its expected value. Comparisons of the dosimetric parameters between post-operative verification and preoperative plans were made by paired $t$-test. One-way ANOVA model was used to assess the differences in preoperative plans. Agreements were evaluated between the preoperative planning and post-operative actual dose parameters using Bland-Altman analysis.

Results: In total, 825 of ${ }^{125}$ I seeds were implanted successfully in 15 patients. In TRUS-based dosimetry, 674 seeds $(81 \%)$ were identified clearly in TRUS-based images, and the expected value of $\mathrm{D}_{90}$ parameter showed no significant differences compared with the preoperative planning and CT post-operation results $(p>0.05)$. In CT-based dosimetry, 810 seeds $(98 \%)$ were identified clearly in CT-based images, and there was good consistency of $\mathrm{D}_{90}, \mathrm{~V}_{100}$, and $\mathrm{V}_{200}$ values $(p>0.05)$. Post-implant CT-based dosimetry indicated that ${ }^{125} \mathrm{I}$ seed implantation had fulfilled the expected plan.

Conclusions: Intraoperative TRUS can be used for dosimetric verification of BT for prostate cancer.
\end{abstract}

Key words: prostate cancer, brachytherapy, TRUS, CT, dosimetry, iodine- 125 .

\section{Purpose}

Brachytherapy (BT) is a standard treatment for patients with localized prostate cancer. BT monotherapy is regarded as a highly efficacious and convenient treatment option for low- to intermediate-risk prostate cancer. It is safe, minimally invasive, and effective, with few complications, produces low radiation pollution, and offers convenient protection, among other advantages over other treatment options $[1,2]$. Post-implant evaluation is essential for the quality assurance of permanent radioactive seed implants in BT [3]. The efficacy of BT using radioactive seeds depends on several procedural factors, such as transrectal ultrasound (TRUS) imaging, magnetic resonance imaging (MRI), computed tomography (CT) quality, surgical skill of clinicians, placement of needles at desired positions, seeds' deposition at planned location, and prevention of seed movement or migration [4]. The biological effect produced by radiotherapy is directly determined by the dose of absorbed radiation by tumor tissue. The actual radiation dose of the tumor and surrounding organs is the most direct and important factor affecting therapeutic effects and complications of BT. Post-implant dosimetry is the gold standard in the assurance of implant quality. Moreover, the implant quality of seeds can be assessed by performing dosimetry with parameters, such as $\mathrm{D}_{90}, \mathrm{~V}_{100}$, and $\mathrm{V}_{200}$ of whole prostate for analysis [5].

At present, CT-based dosimetry is recommended as it can display the prostate as well as the seeds [6]. However, in CT-based dosimetry, seed implant adjustment cannot be performed unless a second surgery is performed. 
In addition, CT-based dosimetry has been found to be insufficient, and the prostate edge is difficult to identify, particularly because of the interference of artifacts produced by implanted seeds. In this case, TRUS-based dosimetry might be an alternative method. There is a small number of reports comparing the consistency between TRUS-based and CT-based dosimetry, and few reports have ever studied intraoperative TRUS-based dosimetry of BT for prostate cancer $[7,8,9,10]$. The purpose of this study was to investigate the possibility of intraoperative TRUS-based dosimetry of BT for prostate cancer.

\section{Material and methods}

\section{Patients' characteristics}

This study was approved by the ethic committee of Xiangya Hospital, and all a written consent was obtained from every patient. All patients underwent an evaluation before BT, including a detailed history, physical examination, pelvic CT or MRI imaging, chest X-ray, abdominal ultrasonography, prostate biopsy, and bone scanning. Eligibility criteria were as follows: clinical tumor stage $\mathrm{T}_{1}$ or $\mathrm{T}_{2}$, Gleason score $<8$, prostate specific antigen (PSA) $<20 \mathrm{ng} / \mathrm{ml}$, and no evidence of gross extracapsular extension, regional node involvement, or metastatic disease. Exclusion criteria included excessively large prostate volume $\left(>60 \mathrm{~cm}^{3}\right)$, history of transurethral prostatectomy, history of pelvic radiotherapy or multiple pelvic surgeries, severe diabetes and other systemic diseases, history of neoadjuvant androgen deprivation therapy, or prominent middle zone of prostate [11]. The preimplant CT or MRI imaging data were used to evaluate the risk of potential interference with needle insertion by the pubic bone.

\section{Preimplant plan}

The preimplant plan was carried out 1 week before seed implantation in the treatment planning system (TPS, Prowess Panther Brachy version 5.0) with the patients in the dorsal lithotomy position under TRUS guidance. Transverse images of the prostate from base to apex were scanned with a $5 \mathrm{~mm}$ slice thickness, and all collected images were imported into the TPS. Axial and sagittal ultrasound images of the prostate were reconstructed by the TPS. After setting tumor matching peripheral dose (MPD) and analyzing maximum tolerance doses of important tissues and organs around the target area, the proper positioning of iodine-125 $\left({ }^{125} \mathrm{I}\right)$ radioactive seeds was designed. The minimal peripheral dose (MPD) was prescribed as 145 Gy (TG43) and was planned to cover $99 \%$ or more of the PTV, with a 5-mm margin in all directions except posteriorly, where the margin was restricted to 2 or $3 \mathrm{~mm}$. According to the standards recommended by the American Brachytherapy Society, the MPD was established as $145 \mathrm{~Gy}$, with limitations in periurethral tissue $\mathrm{D}_{5}$ to $<150 \mathrm{~Gy}$ and in rectum $\mathrm{D}_{1 \mathrm{cc}}$ to $<145 \mathrm{~Gy}$ [12].

\section{Implant procedure}

Cross-sectional images of the prostate were taken by TRUS (HI VISION Avius, Hitachi, Japan) and imported into the TPS. An intraoperative implant plan was produced again instantly before seed implantation. A prescribed dose was planned to cover over $95 \%$ of the target volume. Seed implants were carried out under general anesthesia. The needles containing ${ }^{125}$ I seeds (6711 model, CZB Health, China) with an apparent activity of $0.4 \mathrm{mCi}$ and an air kerma strength of $0.508 \mathrm{U}\left(\mu \mathrm{Gy} \mathrm{m}^{2} \cdot \mathrm{h}^{-1}\right)$ were used, and the seeds were inserted into the prostate transperineally through a template for guidance. The seeds were implanted one-by-one at the target point through the needles using a Mick applicator (Figure 1).

\section{Postimplant dosimetry}

The appearance and characteristics of the seeds in intraoperative TRUS images were divided into four categories, according to Jamaluddin et al. (Figure 2A): a) Definitely no seed, b) Likely no seed, c) Likely seed, d) Definitely seed [13]. In dosimetry, post-implant $D_{90}$,
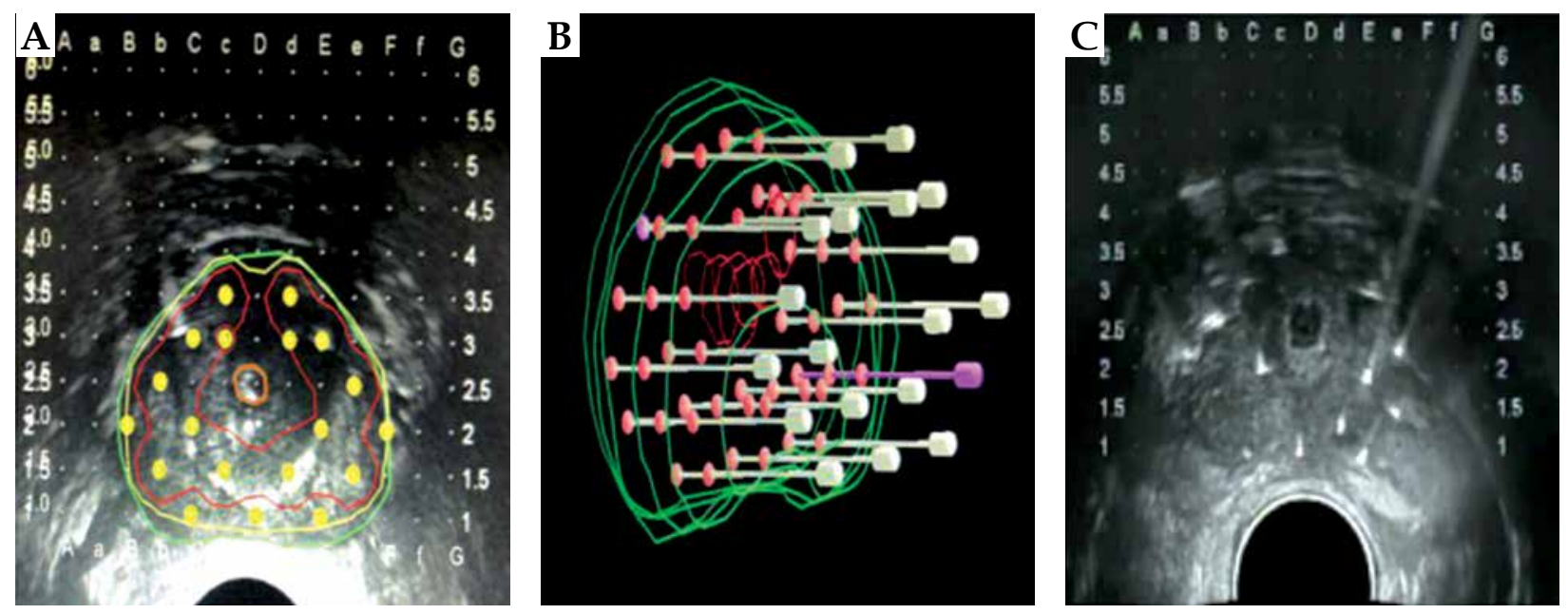

Fig. 1. A) Preimplant radiotherapy plan (a series of axial images was imported into treatment planning system [TPS], with prostate gland, and urethra structures reconstructed using the contouring tool), B) Contour of preplant plan in TPS, C) Intraoperative TRUS images 

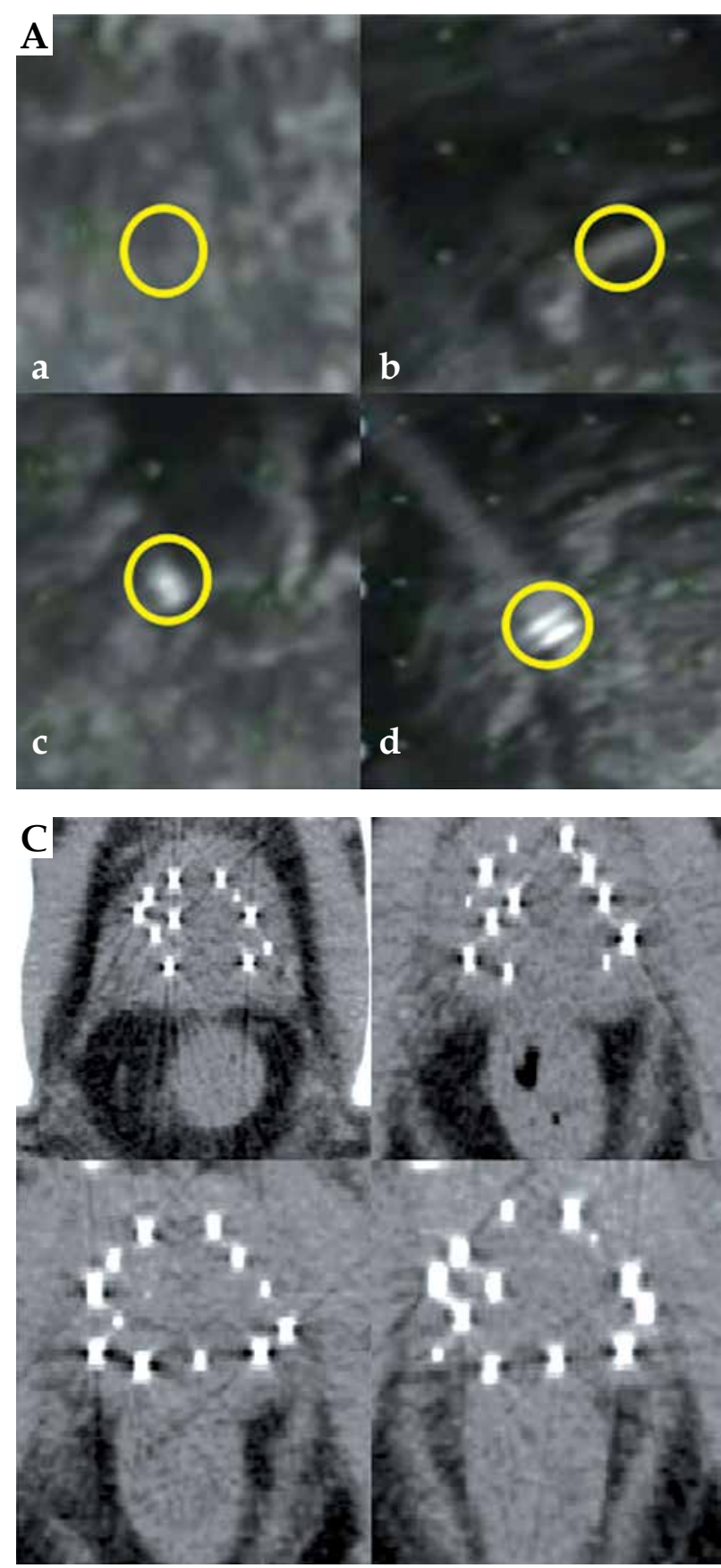

$\mathrm{V}_{100}$, and $\mathrm{V}_{200}$ values were calculated by analyzing the intraoperative TRUS images imported into the TPS.

The ultrasound images of the prostate from base to apex were scanned again immediately after the procedure on the surgical bed and imported into the TPS to immediately evaluate the dosimetry. In post-implant dosimetry, post-implant axial CT images of the pelvis were completed using a CT scanner (SOMATOM Definition, Siemens, Germany) at 3.0-mm slice thickness and 2.5-mm slice spacing, with the patient in supine position at the post-anesthesia care unit. The CT images were also imported into the TPS to evaluate the dosimetry (Figure 2C).

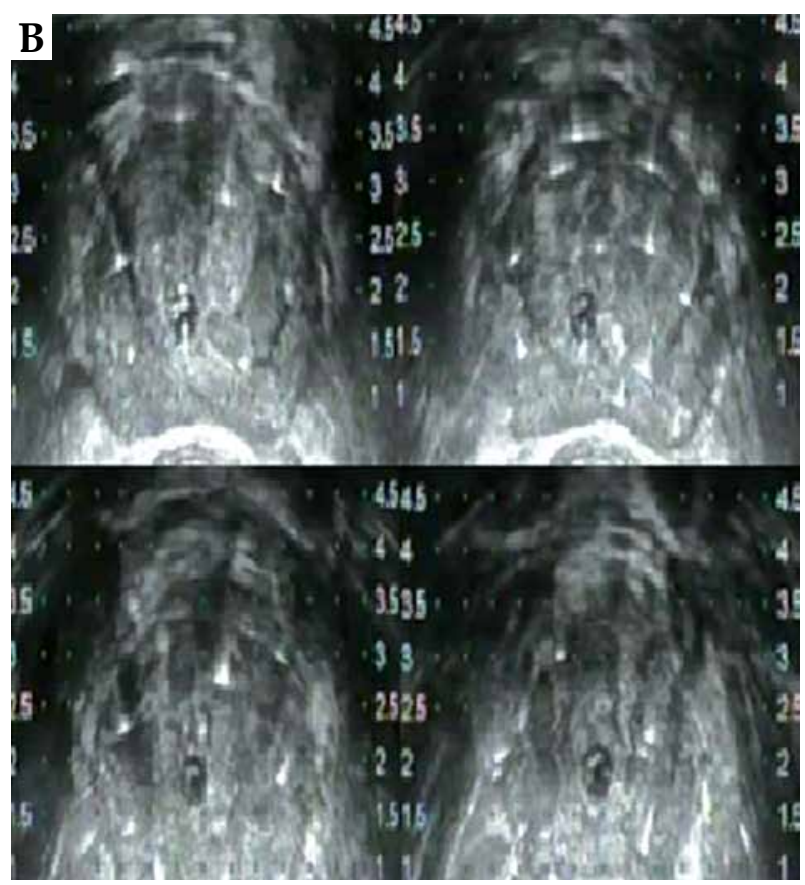

Fig. 2. A) TRUS-based identification of implanted seeds (a. Definitely no seed, b. Likely no seed [only needle tracks are seen as slightly higher echogenicity from the background], c. Likely seed [higher intensity echogenicity], d. Definite seed [higher intensity echogenicity coupled with the "comet tail" artifact]), B) TRUS images of seeds (different levels of ultrasound images were intercepted and imported into treatment planning system [TPS]), C) Post-implant CT images of seeds

\section{Statistical analyses}

Application of SPSS version 18.0 was used for statistical analysis and the data was presented as the means \pm standard deviations. $P<0.05$ was considered statistically significant. Comparisons of the dosimetric parameters between post-operative verification and preoperative plans were made using a paired $t$-test, and dose parameters included $\mathrm{D}_{90}, \mathrm{~V}_{100}$, and $\mathrm{V}_{200}$. One-way ANOVA was applied to assess the differences in preoperative plans, and in the TRUS-based and CT-based dosimetry. Data was normally distributed when $t$-test and ANOVA was performed in SPSS. An agreement was evaluated between the preoperative planning and post-operative actual dose parameters 
using Bland-Altman analysis. The analysis was also carried out using SPSS version 18.0.

\section{Results}

\section{Patient characteristics}

Fifteen patients consented to participate in this study. The average age of patients was 66 years (range, 59-81 years). The preimplant ultrasound average prostate volume was $49 \mathrm{~cm}^{3}$ (range, 29-60 $\mathrm{cm}^{3}$ ). Three patients had low-risk prostate cancer, and 12 patients had intermediate-risk prostate cancer. The characteristics of patients are summarized in Table 1.

\section{Seed implant evaluation}

Computed tomography images demonstrated that the ${ }^{125}$ I seed implant had fulfilled the expected plan. In total 825 of ${ }^{125}$ I seeds were implanted successfully within all patients. TRUS-based verification showed highly echogenic

Table 1. Summary of patients and disease characteristics

\begin{tabular}{|c|c|}
\hline \multicolumn{2}{|l|}{ Age (years) } \\
\hline Mean (range) & $66(59-81)$ \\
\hline \multicolumn{2}{|l|}{ Prostate volume (ml) } \\
\hline Mean (range) & $49(26-60)$ \\
\hline \multicolumn{2}{|l|}{ PSA (ng/ml) } \\
\hline Mean (range) & $14.01(1.08-19.60)$ \\
\hline \multicolumn{2}{|l|}{ Clinical T classification } \\
\hline T1c & 2 \\
\hline T2a & 10 \\
\hline $\mathrm{T} 2 \mathrm{~b}$ & 1 \\
\hline T2c & 2 \\
\hline \multicolumn{2}{|l|}{ Gleason score } \\
\hline $3+3$ & 3 \\
\hline $3+4$ & 3 \\
\hline $4+3$ & 9 \\
\hline \multicolumn{2}{|l|}{ Risk category } \\
\hline Low & 3 \\
\hline Intermediate & 12 \\
\hline \multicolumn{2}{|l|}{ Needles } \\
\hline$N$ & 314 \\
\hline Mean per patient (range) & $21(14-33)$ \\
\hline \multicolumn{2}{|l|}{ Sources } \\
\hline$N$ & 825 \\
\hline Mean per patient (range) & $54(42-86)$ \\
\hline
\end{tabular}

areas on the ultrasound, which corresponded with "Likely seed" or "Definitely seed" (Figure 2A,C,D). Therefore, 674 seeds were clearly identified and included in the analysis. The identification rate of seeds in the TRUS-based images was approximately $81 \%$. In the CT-based verification, 810 seeds were identified. The identification rate of seeds in the CT images was approximately $98 \%$.

\section{Dosimetry}

The $\mathrm{D}_{90}, \mathrm{~V}_{100}$, and $\mathrm{V}_{200}$ dosimetry parameters from the preimplant plan and post-implant CT-based and TRUSbased verifications for the 15 patients are listed and summarized in Tables 2-4. Post-implant CT-based dosimetry indicated that ${ }^{125} \mathrm{I}$ seed implantation had fulfilled the expected plan (Table 5). The comparison of preimplant planned and post-implant dosimetric parameter $\mathrm{D}_{90}$ of the prostate showed no significant differences between TRUS-based and CT-based dosimetry $(p>0.05)$. One-way ANOVA showed no significant differences among the preoperative plans and the TRUS-based and CT-based dosimetries (Table 6). In the CT-based dosimetry, there was good consistency among $\mathrm{D}_{90}, \mathrm{~V}_{100}$, and $\mathrm{V}_{200}$ (Figure $3 \mathrm{~A}-\mathrm{C})$. Figure 3D indicates good consistency between the expected value of TRUS-based and actual CT-based dosimetry in $\mathrm{D}_{90}$.

\section{Discussion}

Low-dose-rate brachytherapy allows for a higher therapeutic radiation dose to the prostate tumor with a steep dose gradient to surrounding normal tissues, resulting in less damage to surrounding normal tissue, fewer complications, mild pain, accurate curative effects, rapid post-operative recovery, and easy acceptance of patients $[2,14]$. The therapeutic effects of radioactive seeds are directly dependent on dose distribution in the target area. The accuracy of radioactive seed placement is important for optimal dose delivery to the prostate gland, while sparing organs at risk [15]. A preimplant plan is needed to design expected dose distribution within the target area before implantation. Also, post-implant dosimetry is essential to acquire appropriate number and strength of sources, which are considerably important to ensure clinical efficacy and safety by evaluating curative effects, determining the potential need to replant seeds, and providing scientific data for the analysis of dose-effect relationships. A major factor interrupting the concordance between the intended and actual implant results is seed placement accuracy, which is greatly affected by proper needle insertion. Other factors determine the accuracy of needle placement, such as the therapist's skills level, imaging, and equipment quality as well as the degree of prostate immobilization and mobility. A study by Prestidge $e t$ al. evaluated the timing of CT-based post-implant assessment following permanent transperineal prostate brachytherapy, and showed that scans performed at $30^{\text {th }}$ post-implant day appear to adequately describe the time-averaged dose coverage of the prostate [16]. It is best to conduct dosimetry immediately or 1 month after the procedure. As recommended, the implant quality of BT 
Table 2. Preimplant and post-implant dosimetric parameters in target volume of 15 patients

\begin{tabular}{lccccccccc} 
No. & \multicolumn{3}{c}{$\mathrm{D}_{90}[\mathrm{~Gy}]$} & \multicolumn{3}{c}{$\mathrm{V}_{100}[\%]$} & \multicolumn{3}{c}{$\mathrm{V}_{200}[\%]$} \\
\cline { 2 - 11 } & Pre & TRUS & Post-CT & Pre & TRUS & Post-CT & Pre & TRUS & Post-CT \\
\hline 1 & 146.2 & 120.8 & 143.4 & 98.2 & 85.4 & 94.6 & 54.5 & 40.5 & 54.3 \\
\hline 2 & 145.8 & 115.5 & 140.6 & 96.9 & 81.5 & 95.0 & 54.2 & 41.3 & 53.2 \\
\hline 3 & 147.0 & 119.8 & 151.2 & 95.9 & 83.3 & 94.9 & 55.5 & 41.2 & 55.3 \\
\hline 4 & 146.9 & 122.5 & 153.6 & 98.2 & 86.3 & 94.0 & 53.4 & 40.7 & 54.2 \\
\hline 5 & 147.7 & 123.2 & 146.3 & 95.3 & 89.3 & 93.4 & 55.0 & 40.9 & 54.9 \\
\hline 6 & 148.6 & 126.5 & 144.5 & 95.9 & 81.3 & 92.7 & 53.7 & 39.7 & 55.4 \\
\hline 7 & 145.2 & 117.3 & 154.3 & 95.8 & 85.8 & 92.5 & 55.3 & 41.1 & 55.0 \\
\hline 8 & 149.6 & 120.0 & 145.2 & 98.9 & 83.0 & 97.9 & 54.9 & 41.5 & 52.7 \\
\hline 9 & 148.2 & 116.2 & 140.5 & 96.1 & 83.1 & 94.1 & 53.8 & 40.0 & 52.9 \\
\hline 10 & 145.1 & 124.7 & 150.8 & 95.8 & 85.4 & 94.0 & 54.0 & 40.3 & 53.8 \\
\hline 11 & 146.3 & 129.8 & 156.6 & 99.5 & 87.8 & 96.0 & 54.1 & 40.6 & 56.2 \\
\hline 12 & 149.3 & 126.2 & 153.0 & 96.5 & 85.6 & 95.7 & 54.8 & 40.7 & 55.9 \\
\hline 13 & 148.7 & 117.7 & 142.4 & 96.6 & 83.1 & 93.6 & 54.4 & 41.0 & 54.1 \\
\hline 14 & 147.5 & 123.0 & 145.6 & 95.5 & 88.7 & 94.3 & 54.9 & 41.1 & 55.0 \\
\hline 15 & 148.4 & 120.6 & 152.3 & 96.8 & 87.1 & 96.5 & 54.7 & 41.4 & 53.4
\end{tabular}

Pre - preimplant plan, TRUS - TRUS-based, Post-CT - post-implant CT-based, $D_{90}$ - dose received by $90 \%$ of the prostate volume, $V_{100}, V_{200}-$ percentage of the prostate receiving $100 \%$ and $200 \%$ of the prescribed radiation dose

can be assessed by performing post-implant CT-based dosimetry [17]. In present study, we used Bland-Altman analysis to show the similarity between pre-implant TRUS plan and post-implant CT, and to prove the accuracy of preimplant plan of TRUS. However, the validity of TRUS-based dosimetry during BT procedure for prostate cancer remains to be evaluated.

In this study, 825 of ${ }^{125} \mathrm{I}$ seeds were implanted successfully in 15 patients from our center, and 810 seeds (98\%) were verified by post-implant CT images. We considered the possibility of inter-seed effect before the study. Safigholi et al. [18] believed that this effect might influence dosimetry planning and validation. However, we found that the effect was not so dramatic in the CTbased dosimetry, because there was a good consistency among $\mathrm{D}_{90}, \mathrm{~V}_{100}$, and $\mathrm{V}_{200}$. The consistency of $\mathrm{D}_{90}, \mathrm{~V}_{100}$, and $V_{200}$ values indicated that the ${ }^{125}$ I seed implant had fulfilled the expected plans. Meanwhile, TRUS-based seed verification showed that 674 seeds $(81 \%)$ could be clearly identified in the TRUS images, demonstrating that the unclear visualization of seeds in TRUS remains a major challenge. After the $\mathrm{D}_{90}$ value from the TRUSbased dosimetry was transformed to the expected value, we compared the dosimetric parameters between the post-operative verification and preoperative plans. The expected value of the $D_{90}$ parameter showed no
Table 3. The $\mathrm{D}_{90}$ dosimetry parameter of TRUS expected value

\begin{tabular}{lccc} 
No. & \multicolumn{3}{c}{$\mathrm{D}_{90}[\mathrm{~Gy}]$} \\
\cline { 2 - 4 } & TRUS & Percentage & Expected value \\
\hline 1 & 120.8 & 0.83 & 145.5 \\
\hline 2 & 115.5 & 0.81 & 142.5 \\
\hline 3 & 119.8 & 0.79 & 151.7 \\
\hline 4 & 122.5 & 0.82 & 149.3 \\
\hline 5 & 123.2 & 0.84 & 146.7 \\
\hline 6 & 126.5 & 0.83 & 152.4 \\
\hline 7 & 117.3 & 0.78 & 150.5 \\
\hline 8 & 120.0 & 0.82 & 146.3 \\
\hline 9 & 116.2 & 0.79 & 147.1 \\
\hline 10 & 124.7 & 0.83 & 150.2 \\
\hline 11 & 129.8 & 0.85 & 151.4 \\
\hline 12 & 126.2 & 0.84 & 150.3 \\
\hline 13 & 117.7 & 0.80 & 147.1 \\
\hline 14 & 123.0 & 0.81 & 151.8 \\
\hline 15 & 120.6 & 0.83 & 145.3
\end{tabular}

Percentage - percentage of seeds identified, expected value - TRUS/percentage of seeds identified

Table 4. Summary of preimplant planned dosimetry and post-implant dosimetry

\begin{tabular}{lccc} 
& $\mathrm{D}_{90}[\mathrm{~Gy}]$ & $\mathrm{V}_{100}[\%]$ & $\mathrm{V}_{200}[\%]$ \\
\hline Preimplant planned & $147.37 \pm 1.44$ & $96.79 \pm 1.30$ & $54.52 \pm 0.49$ \\
\hline TRUS-based & $123.52 \pm 5.62$ & $85.11 \pm 2.49$ & $40.78 \pm 0.49$ \\
\hline TRUS-based expected value & $150.07 \pm 4.71$ & - & - \\
\hline Postimplant CT-based & $148.02 \pm 5.33$ & $95.86 \pm 1.66$ & $53.40 \pm 0.83$
\end{tabular}


Table 5. Comparison of preimplant planned and post-implant CT-based dosimetric parameter of the prostate

\begin{tabular}{lccc} 
& $\mathrm{D}_{90}$ & $\mathrm{~V}_{100}$ & $\mathrm{~V}_{200}$ \\
\hline Preimplant planned & $147.37 \pm 1.44$ & $96.79 \pm 1.30$ & $54.52 \pm 0.49$ \\
\hline Post-procedure CT-based & $148.02 \pm 5.33$ & $95.86 \pm 1.66$ & $53.40 \pm 0.83$ \\
\hline$T$-value & -0.43 & 1.69 & 0.52 \\
\hline$P$-value & 0.67 & 0.11 & 0.61
\end{tabular}

Table 6. Comparison of preimplant planned and post-implant $\mathrm{D}_{90}$ dosimetric parameter of the prostate

\begin{tabular}{llc} 
Group & & $\mathrm{D}_{90}$ \\
\hline A & $\begin{array}{c}\text { Preimplant } \\
\text { planned }\end{array}$ & $147.37 \pm 1.44$ \\
\hline B & $\begin{array}{c}\text { Post-implant } \\
\text { TRUS-based } \\
\text { expected value }\end{array}$ & $150.07 \pm 4.71$ \\
\hline C & $\begin{array}{c}\text { Post-procedure } \\
\text { CT-based }\end{array}$ & $148.02 \pm 5.33$ \\
\hline One-way ANOVA: $F, p$ & A vs. B & $0.39,0.67$ \\
\hline T-test: $t, p$ & A vs. C & $-1.3,0.19$ \\
\cline { 2 - 3 } & B vs. C & $0.43,0.67$ \\
\cline { 2 - 3 } & &
\end{tabular}

significant differences compared with the preoperative planning and CT post-operative values. This result indicates that ultrasound images can be used for prostate brachytherapy post-implant assessment. We used Bland-Altman analysis to demonstrate the similarity between pre-implant TRUS plan and post-implant CT, and to prove the accuracy of preimplant plan of TRUS (Figure $3 \mathrm{~A}-\mathrm{C})$. As the mean increased, more and more seeds had improved the spatial structure, which reduced differences. The number of seeds detected by post-implant TRUS was less than that of post-implant CT. Therefore, the expected $D_{90}$ in post-implant TRUS was compared with post-implant CT as shown in Figure 3D. The consistency was satisfactory. Therefore, if the $\mathrm{D}_{90}$ value of the TRUSbased dosimetry was transformed to the expected value, dosimetry assessments could be performed between ultrasound and CT verification. This approach is an exploratory attempt, underlying assumption that each seed contributes linearly to the $\mathrm{D}_{90}$. It is not the most accurate and ideal method to calculate the ratio of seeds found by intraoperative ultrasound, but this calculation considers the proportion of seeds found. At the same time, the influence of spatial distributions can mainly be reflected in an intraoperative ultrasound dose verification, although it cannot be accurately calculated.

We performed CT scans and ultrasound immediately after the procedure, and the TRUS prostate volume showed a minor difference when compared with the preimplant ultrasound volume for each patient. The CT scan-determined volume was slightly greater than the volume determined by the preimplant ultrasound.
It might be that prostate enlargements are not be visible immediately after the surgery.

In clinical practice, TRUS-based dosimetry has some important advantages over CT-based dosimetry. CTbased dosimetry cannot be used to compare the locations of implanted seeds with those from a preimplant plan, because of changes in body position in post-implant CT images. Also, post-implant CT cannot readily allow for adjusting seed implants once they are established. In contrast, intraoperative TRUS-based dosimetry cannot only be carried out in real time but allows seed implant adjustment when real-time dosimetry is found to be insufficient. Moreover, the implant procedure is very safe, as the patient is in a constant position during the procedure, and most notably, the prostate and urethra are well visualized in TRUS images. Additionally, needle placement can be followed in real-time during insertion, which allows the adjustment of subsequent needle positions to compensate for any improper needle placement [19]. Overall, TRUSbased dosimetry is reliable and sufficiently flexible for intraoperative dosimetry evaluation.

There are some limitations of this study. First, the number of patients was small, which limits the statistical significance of the outcomes. Second, this study was a single-center experience, not a prospective multicenter research. Additionally, TRUS-based source identification presents the disadvantage of low sensitivity because of relatively poor tissue contrast resolution, estimated between $51 \%$ and $83 \%$ [20]. The results show that the poor visualization of seeds in TRUS remains a major challenge. The main goal of the present study was to explore the possibility of utilizing single ultrasound-base dosimetry during the procedure. However, only a majority $(81 \%)$ of the seeds could be observed by ultrasound due to the limitation of image quality. We could speculate that "majority" or " $81 \%$ " is sufficient for a single ultrasound-base dosimetry, and the general distribution of seeds could be guaranteed according to the detection of ultrasound. Obviously, a single ultrasound-base dosimetry needs large and multicenter study to confirm its validity. It is possible that seed identification could be improved with various methods, such as addition of sagittal imaging, which could enhance visualization. Moreover, using a 3-dimensional view of the needle tracks and its interpolation capability could enhance seed identification rate. The quality of images is also critical for dosimetric analysis. Therefore, we hypothesized that it is possible that real-time intraoperative seed identification could be more precise with future technological improvements. 
A

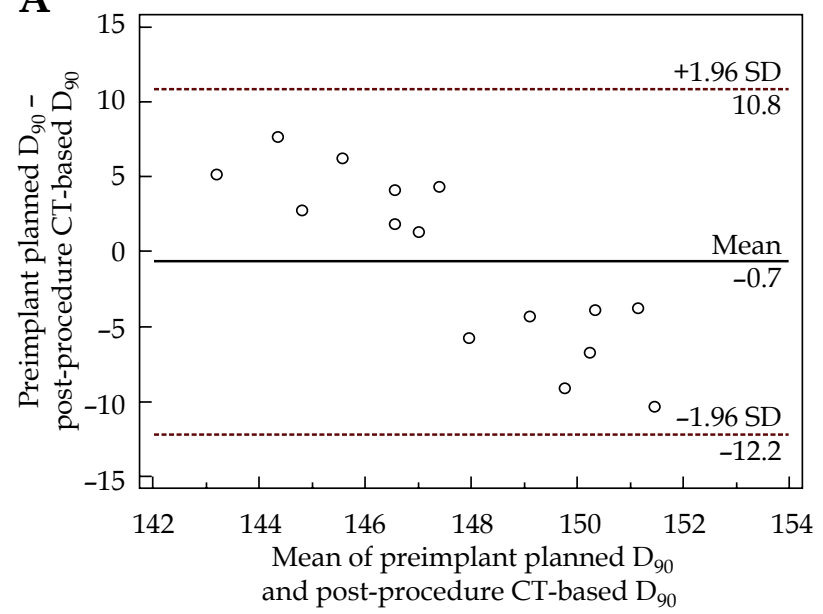

C

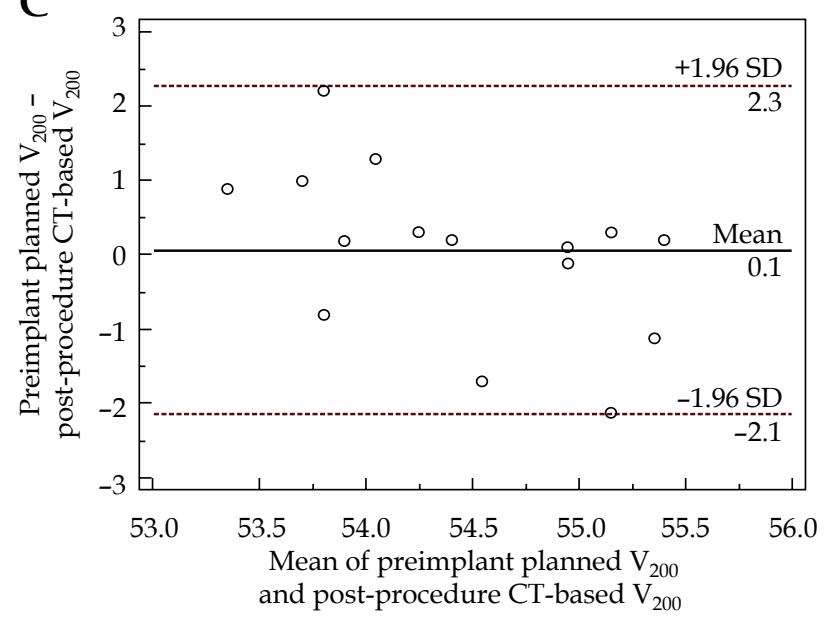

B

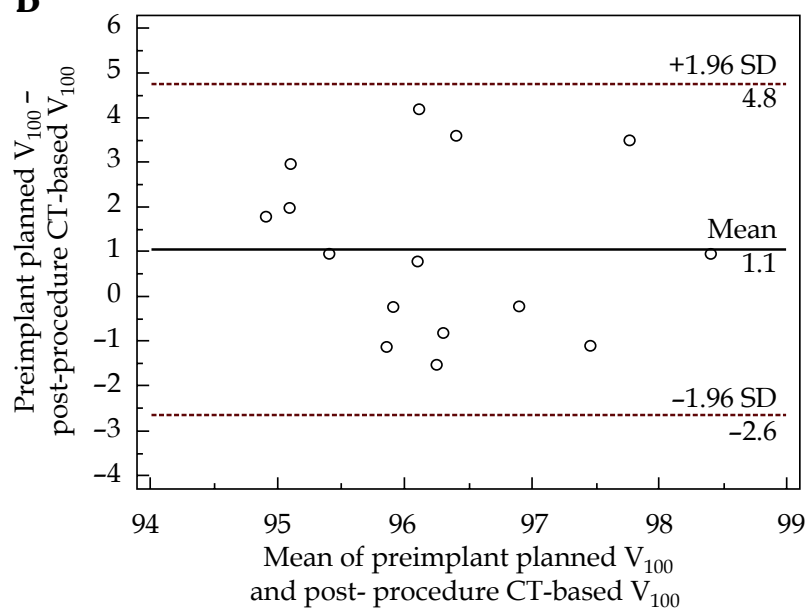

D

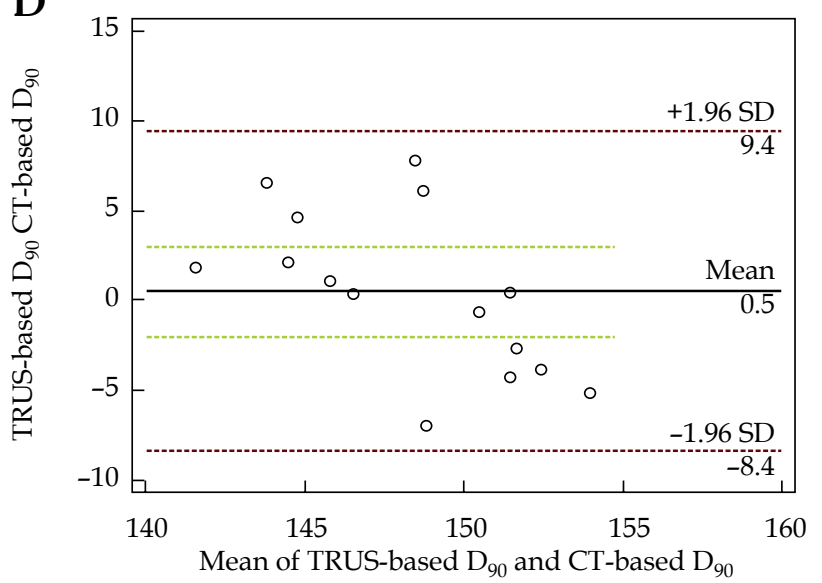

Fig. 3. Bland-Altman plot showing the scale from the average of preimplant plan to dosimetry parameters with limits of agreement (LoA) (broken lines). Bland-Altman plot of the difference between preimplant and post-implant of CT-based $\mathrm{D}_{90}$ (A), $\mathrm{V}_{100}(\mathbf{B})$, and $\mathrm{V}_{200}(\mathbf{C})$. Figure D shows good consistency between expected value of TRUS-based and actual CT-based dosimetry in $\mathrm{D}_{90}$

\section{Conclusions}

The present study indicates that a single TRUS-based dosimetry can be used for a post-implant assessment of prostate brachytherapy. Compared with CT-based dosimetry, TRUS-based dosimetry can be conveniently performed in real-time. In addition to CT, TRUS is a valuable dosimetry method in prostate cancer brachytherapy.

\section{Acknowledgments}

This work was supported by the following grants: the National Key R\&D Program of China (No. 2017YFC0908004), the National Natural Science Foundation of China (No. 81874094), the Hunan Provincial Natural Science Foundation of China (No. 2019JJ40484), the Science and Technology Plan Projects of Changsha City (No. kq1801114), the Project from Health and Family Planning Commission of Hunan Province (No. C20180105), and the Innovation and Entrepreneurship in South Central University project (No. 2017gczd032).

\section{Disclosure}

The authors report no conflict of interest.

\section{References}

1. Chin J, Rumble RB1, Kollmeier M et al. Brachytherapy for patients with prostate cancer: American Society of Clinical Oncology/Cancer Care Ontario Joint Guideline Update. J Clin Oncol 2017; 35: 1737-1743.

2. Pickles T, Keyes M, Morris WJ. Brachytherapy or conformal external radiotherapy for prostate cancer: A single-institution matched-pair analysis. Int J Radiat Oncol Biol Phys 2010; 76: 43-49.

3. Bowes D, Crook JM, Araujo C et al. Ultrasound-CT fusion compared with MR-CT fusion for postimplant dosimetry in permanent prostate brachytherapy. Brachytherapy 2013; 12: 38-43.

4. Podder $\mathrm{T}$, Song $\mathrm{D}$, Showalter $\mathrm{T}$ et al. Advances in radiotherapy for prostate cancer treatment. Prostate Cancer 2016; 2016: 3079684.

5. Okazaki E, Kuratsukuri K, Ishii K et al. Correlations of post-implant regional dosimetric parameters at 24 hours and one month, with clinical results of low-dose-rate brachytherapy for localized prostate cancer. J Contemp Brachytherapy 2017; 9: 499-507.

6. Nag S, Bice W, DeWyngaert $\mathrm{K}$ et al. The American Brachytherapy Society recommendations for permanent prostate brachytherapy postimplant dosimetric analysis. Int J Radiat Oncol Biol Phys 2000; 46: 221-230. 
7. Peinemann F, Grouven U, Hemkens LG et al. Low-dose rate brachytherapy for men with localized prostate cancer. Cochrane Database Syst Rev 2011; 7: CD008871.

8. Shiraishi Y, Yorozu A, Ohashi T et al. A dose-response analysis of biochemical control outcomes after (125)I monotherapy for patients with favorable-risk prostate cancer. Int J Radiat Oncol Biol Phys 2014; 90: 1069-1075.

9. Bice WS Jr, Prestidge BR, Sarosdy MF. Sector analysis of prostate implants. Med Phys 2001; 28: 2561-2567.

10. Ishiyama H, Sekiguchi A, Satoh T et al. Dosimetry of permanent interstitial prostate brachytherapy for an interoperative procedure, using O-arm based CT and TRUS. J Contemp Brachytherapy 2016; 8: 7-16.

11. Mottet N, Bellmunt J, Bolla M et al. EAU-ESTRO-SIOG Guidelines on Prostate Cancer. Part 1: Screening, diagnosis, and local treatment with curative intent. Eur Urol 2017; 71: 618-629.

12. Davis BJ, Horwitz EM, Lee WR et al. American Brachytherapy Society consensus guidelines for transrectal ultrasound-guided permanent prostate brachytherapy. Brachytherapy 2012; 11: 6-19.

13. Jamaluddin MF, Ghosh S, Waine MP et al. Quantifying 125I placement accuracy in prostate brachytherapy using postimplant transrectal ultrasound images. Brachytherapy 2017; 16: 306-312.

14. Wang J, Wang J, Liao A et al. The direct biologic effects of radioactive 125I seeds on pancreatic cancer cells PANC-1, at continuous low-dose rates. Cancer Biother Radiopharm 2009; 24: 409-416.

15. Stock RG, Stone NN. Importance of post-implant dosimetry in permanent prostate brachytherapy. Eur Urol 2002; 41: 434-439.

16. Prestidge BR, Bice WS, Kiefer EJ et al. Timing of computed tomography-based postimplant assessment following permanent transperineal prostate brachytherapy. Int J Radiat Oncol Biol Phys 1998; 40: 1111-1115.

17. Taussky D, Igidbashian L, Donath $\mathrm{D}$ et al. Is intraoperative real-time dosimetry in prostate seed brachytherapy predictive of biochemical outcome? J Contemp Brachytherapy 2017; 9: 304-308.

18. Safigholi H, Sardari D, Karimi Jashni S et al. An analytical model to determine interseed attenuation effect in low-doserate brachytherapy. J Appl Clin Med Phys 2013; 14: 4226.

19. Schmid M, Crook JM, Batchelar D et al. A phantom study to assess accuracy of needle identification in real-time planning of ultrasound-guided high-dose-rate prostate implants. Brachytherapy 2013; 12: 56-64.

20. Han BH, Wallner K, Merrick G et al. Prostate brachytherapy seed identification on post-implant TRUS images. Med Phys 2003; 30: 898-900. 